\title{
EIGENFUNCTIONS OF THE LAPLACIAN ON COMPACT RIEMANNIAN MANIFOLDS*
}

\author{
HAROLD DONNELLY ${ }^{\dagger}$
}

Key words. Mathematical physics, quantum chaos, ergodic geodesic flow

AMS subject classifications. $58 \mathrm{G} 25$

0. Introduction. Suppose that $M$ is a compact Riemannian manifold. The Laplace operator $\Delta$ is given in local coordinates by $\Delta f=g^{-1 / 2} \frac{\partial}{\partial x_{i}}\left(g^{1 / 2} g^{i j} \frac{\partial f}{\partial x_{j}}\right)$, where $g_{i j}$ denotes the metric tensor and $g=\operatorname{det}\left(g_{i j}\right)$. It follows from the compactness of $M$ and the theory of elliptic partial differential equations that $\Delta$ has pure point spectrum. This means that $L^{2} M$ admits an orthonormal basis consisting of eigenfunctions $\phi_{i}$ of $\Delta$ with associated eigenvalues $\lambda_{i}$, that is $\Delta \phi_{i}=-\lambda_{i} \phi_{i}$. There have been many works concerning the eigenvalues and their relationship to the geometry of the manifold. These studies pertain to upper and lower bounds for eigenvalues and asymptotics of eigenvalues. The research concerning the eigenfunctions themselves is less highly developed.

The present paper constitutes an exposition of some topics of interest in current research about eigenfunctions. One source of inspiration is the mathematical physics of quantum chaos, which is concerned with the quantization of classically ergodic systems. The first two sections of the paper discuss concentration properties of eigenfunctions. Concentration is measured both through bounds of the supremum norm and via weak limits of the associated densities $\left|\phi_{i}\right|^{2} d v o l$, as $i \rightarrow \infty$. Theorem 2.3 is a fundamental result concerning the weak limits of these densities on manifolds with ergodic geodesic flow. This theorem of quantum ergodicity has been studied extensively by several mathematicians. Our third section concerns the nodal sets of eigenfunctions. The main focus is upon the conjecture of Yau concerning the Hausdorff measure of the nodal sets. We include some motivating ideas which may be absent from the more technical presentations appearing in the published record.

This article was solicited by the editors for the memorial volume dedicated to S. S. Chern. It may therefore be appropriate to mention that the author received his doctorate in 1974 under the direction of Professor Chern. The thesis consisted of various results about Chern Simons invariants. However, Professor Chern encouraged his student to learn about other topics of mathematical research. These topics included the study of eigenvalues and eigenfunctions of the Laplace operator for Riemannian manifolds.

1. Bounds for the Supremum Norm of Eigenfunctions. Let $M$ be a compact Riemannian manifold and $\Delta$ its Laplacian acting on functions. Suppose that $\phi$ is an eigenfunction of $-\Delta$ with eigenvalue $\lambda \neq 0, \Delta \phi=-\lambda \phi$. If one scales the metric by $g_{i j} \rightarrow \lambda g_{i j}$, an elliptic equation with bounded coefficients is obtained. Also, a geodesic ball of radius $c \lambda^{-1 / 2}$ scales to a ball of radius $c$. Elementary local elliptic theory shows that the $L^{\infty}$ norm of $\phi$ is bounded by its $L^{2}$ norm relative to the scaled

\footnotetext{
*Received July 7, 2005; accepted for publication November 18, 2005.

$\dagger$ Department of Mathematics, Purdue University, West Lafayette, IN 47907, USA (hgd@math. purdue.edu). Partially supported by NSF Grant 0203070-DMS.
} 
metric. Rescaling back to the original problem yields the estimate

$$
\|\phi\|_{\infty} \leq c_{1} \lambda^{n / 4}\|\phi\|_{2}
$$

where $n$ denotes the dimension of $M$. Elementary examples using exponential functions show that this is the best estimate possible using local considerations only.

Remarkably, Hörmander [16] proved that for eigenfunctions on compact manifolds, one has

$$
\|\phi\|_{\infty} \leq c_{2} \lambda^{(n-1) / 4}\|\phi\|_{2}
$$

The constant $c_{2}$ cannot be scale invariant. In Hörmander's proof, one constructs a parametrix for the fundamental solution of the wave equation. Consequently, $c_{2}$ depends upon $c n$ derivatives of the metric. The paper [16] represents the beginning of the local theory of Fourier Integral Operators. One observes that $(n-1) / 4$ is the optimal power of $\lambda$. In fact, rotationally symmetric spherical harmonics on $S^{n}$ illustrate the sharpness of (1.2).

Besides their intrinsic interest these estimates are closely related to the multiplicity of eigenvalues. Suppose that $E_{\lambda}$ denotes the eigenspace of $-\Delta$ and $m_{\lambda}=\operatorname{dim} E_{\lambda}$. The following elementary consequence of (1.2) is well-known:

LEMMA 1.1. $m_{\lambda} \leq c_{3} \lambda^{(n-1) / 2}$.

Proof. Consider the Bergman kernel $K(x, y)$ for orthogonal projection onto the eigenspace $E_{\lambda}$. If $\phi_{i}$ is any orthonormal basis for $E_{\lambda}$, then $K(x, y)=\sum_{i=1}^{m_{\lambda}} \phi_{i}(x) \phi_{i}(y)$. Since $m_{\lambda}=\int_{M} K(x, x) d v o l_{M}(x)$, there exists at least one point $x_{0} \in M$, where $m_{\lambda} \leq$ $K\left(x_{0}, x_{0}\right) \operatorname{vol}_{M}$. We now choose a special orthonormal basis for $E_{\lambda}$. Let $r_{o}: E_{\lambda} \rightarrow R$ denote the evaluation map at $x_{0}, r_{o}(f)=f\left(x_{0}\right)$. If $\phi_{i} \in \operatorname{Ker}\left(r_{o}\right)$, for all $i \geq 2$, then $K\left(x_{0}, x_{0}\right)=\phi_{1}^{2}\left(x_{0}\right) \leq c_{2}^{2} \lambda^{(n-1) / 2}$, by $(1.2)$.

In [13], the author investigated the geometric dependence of the constant $c_{2}$. Recall that the injectivity radius of $M$ is the radius of the largest ball where exp : $T_{p} M \rightarrow M$ is guaranteed to be a diffeomorphism, for all $p \in M$. One needs a lower bound for the injectivity radius and an upper bound on the absolute value of the sectional curvature. The sectional curvature depends upon two derivatives of the metric only while Hörmander's proof of (1.2) requires $c n$ derivatives of the metric. The complete proof of the next result appears in reference [13]:

THEOREM 1.2. Let $M$ be a compact Riemannian manifold and $\Delta$ its Laplacian acting on functions. Suppose that the injectivity radius of $M$ is bounded below by $c_{4}$ and that the absolute value of the sectional curvature of $M$ is bounded above by $c_{5}$. If $\Delta \phi=-\lambda \phi$ and $\lambda \neq 0$, then $\|\phi\|_{\infty} \leq c_{2} \lambda^{(n-1) / 4}\|\phi\|_{2}$. The constant $c_{2}$ depends only upon $c_{4}, c_{5}$, and the dimension $n$ of $M$. Moreover, the multiplicity $m_{\lambda} \leq c_{3} \lambda^{(n-1) / 2}$, where $c_{3}$ depends only upon $c_{2}$ and an upper bound for the volume of $M$.

Proof. (Sketch) One develops the method of Bessel potentials used by Sogge [21]. Sogge proved (1.2) and more refined $L^{p}$ estimates. Although Sogge did not consider the issue of geometric dependence, his method is more adaptable than the approach of Hörmander.

The Bessel potential $B_{z}(x)$ is the resolvent kernel for $(\Delta+z)^{-1}$, where $\Delta$ denotes the Laplacian of $R^{n}$. The rotational symmetry of the Euclidean metric implies that $B_{z}(x)=B_{z}(r)$, where $r=|x|$. If $r \neq 0$, one clearly has $B_{z}^{\prime \prime}+(n-1) r^{-1} B_{z}^{\prime}+z B_{z}=0$. 
Standard results from ordinary differential equations give asymptotics for $B_{z}$, both for $r \downarrow 0$ and $r \uparrow \infty$.

The rotational symmetry of the Bessel potential allows one to transplant the potential to a Riemannian manifold as a function of the geodesic distance, then $\Delta f(r)=f^{\prime \prime}(r)+\theta^{-1} \frac{\partial \theta}{\partial r} f^{\prime}(r)$, where $\theta(r, w)$ denotes the volume element in geodesic polar coordinates. For $r \leq \min \left(c_{4}, \pi / \sqrt{c_{5}}\right)$, comparison theory [2] gives the bounds $\sqrt{c_{3}}(n-1) \cot \left(\sqrt{c_{3}} r\right) \leq \theta^{-1} \partial \theta / \partial r \leq \sqrt{c_{3}}(n-1) \operatorname{coth}\left(\sqrt{c_{3}} r\right)$. These bounds permit one to estimate the difference between the transplant of the Bessel potential and the resolvent kernel $(\Delta+z)^{-1}$ of $M$.

Since $M$ is compact, $\|\phi\|_{\infty}$ is assumed at some point $p \in M$. Integration by parts gives

$$
|\phi(p)| \leq \sqrt{\lambda}\left|\int_{M} \eta \phi B_{z}\right|+\left|\int_{M} \phi R(x, D) B_{z}\right|
$$

where $\eta$ is a cut-off function and $R(x, D)$ is a first order operator supported in a geodesic ball centered at $p$. One applies this formula with $z=\lambda+i \sqrt{\lambda}$. The proof proceeds through a detailed analysis using the asymptotics of the Bessel potentials.

We now define exceptional sequences of eigenfunctions to be sequences $\phi_{i}$ such that $\left\|\phi_{i}\right\|_{\infty} \geq c \lambda_{i}^{\epsilon}\left\|\phi_{i}\right\|_{2}$, for some $\epsilon>0$. The rotationally symmetric spherical harmonics concentrate on the north and south poles of $S^{n}$, with the maximal value $\epsilon=(n-1) / 4$. However, it is also interesting to consider geometric reasons why intermediate values of $0<\epsilon<(n-1) / 4$ may occur. The author's interest in these questions was first aroused by the following example [4]:

EXAMPLE 1.3. (Bourgain) Let $M=S^{1} \times S^{1}$ be the flat torus. The flat metric may be perturbed to yield a sequence $\phi_{k}$ of eigenfunctions satisfying the following conditions:

a. $\quad \lambda_{k}=k^{2}+0(k)$

b. $\left\|\phi_{k}\right\|_{\infty} \geq c \lambda_{k}^{1 / 8}\left\|\phi_{k}\right\|_{2}$

In [13], we placed the above example within the more general framework of manifolds admitting isometric circle actions. Note that the perturbations constructed by Bourgain exhibit rotational symmetry. Toth and Zelditch [22] study such concentration phenomena for manifolds which are quantum completely integrable. This entails the existence of $n-1$ first order pseudodifferential operators which commute with the Laplacian. Bourgain's example thus fits well into two different but related general schemes.

Suppose that $M$ is any compact manifold admitting an isometric $S^{1}$ action. If $x$ lies in the quotient space $M / S^{1}$, let $2 \pi f(x)$ be the length of the $S^{1}$ orbit lying over $x$. Define $V(x)=f^{-2}(x)$. If $V(x)$ assumes a unique non-degenerate minimum at $p \in$ $M / S^{1}$, then in normal coordinates near $p$, we have $V(x)=V_{\min }+\sum_{i=1}^{n-1} \alpha_{i}^{2} x_{i}^{2}+O\left(|x|^{3}\right)$, where $\alpha_{i}^{2}$ are the eigenvalues of the Hessian of $V / 2$ at $p$, and $\alpha_{i}^{2}>0$ by the nondegeneracy hypothesis. Note that a unique nondegenerate minimum may always be achieved by perturbation of the metric. The complete proof of the next result may be found in [13]:

THEOREM 1.4. Let $M$ be a compact Riemannian manifold with an isometric circle action. Assume that the length of each $S^{1}$ orbit achieves a unique nondegenerate maximum. Then there exists a sequence of eigenfunctions $\phi_{k}$, with eigenvalues $\lambda_{k}$, satisfying: 
a. $\quad \lambda_{k}=k^{2} V_{\min }+k\left(\sum_{i=1}^{n-1} \alpha_{i}\right)+o(k)$

b. $\left\|\phi_{k}\right\|_{\infty} \geq c \lambda_{k}^{\frac{n-1}{8}}\left\|\phi_{k}\right\|_{2}$

Proof. (Sketch) Break $L^{2} M=\oplus L_{k}^{2} M$ into characters of the circle action. The exceptional sequence is formed by choosing the ground states $\phi_{k}$ in each $L_{k}^{2} M$. After separation of variables, the problem is localized near the maximal orbit. The analysis there is modeled upon the simple harmonic oscillator of quantum mechanics:

$$
\begin{aligned}
& -D_{x} \phi_{k}+k^{2}\left(V(x)-V_{\min }\right) \phi_{k}=\left(\lambda_{k}-k^{2} V_{\min }\right) \phi_{k} \\
& V(x)-V_{\min }=\sum \alpha_{i}^{2} x_{i}^{2}+O\left(|x|^{3}\right)
\end{aligned}
$$

$D_{x}$ denotes a second order elliptic operator acting transversely to the circle orbits. From a geometric viewpoint, the basic model is the concentration of eigenfunctions on the equator of $S^{2}$.

In light of the above results, it is natural to ask for which compact Riemannian manifolds one can perturb the metric to yield a sequence of eigenfunctions satisfying $\left\|\phi_{i}\right\|_{\infty} \geq c \lambda_{i}^{\epsilon}\left\|\phi_{i}\right\|_{2}$, for some $\epsilon>0$. Perhaps such perturbation is possible for all manifolds of dimension at least two. In [16], Sarnak conjectured that compact Riemann surfaces of constant negative curvature are exceptional. Note that the isometry group is finite for such Riemann surfaces, so that Theorem 1.4 does not apply. More generally, Sarnak conjectures the following: Let $M$ be a compact Riemann surface with a metric of negative curvature, then all eigenfunctions $\phi$ satisfy a bound $\|\phi\|_{\infty} \leq c_{\epsilon} \lambda^{\epsilon}\|\phi\|_{2}$, for all $\epsilon>0$. If true, Sarnak's conjecture is very deep, because it has intimate connections with the Lindelöf hypothesis in number theory.

Rudnick and Sarnak [18] constructed exceptional sequences of eigenfunction on hyperbolic three manifolds. These sequences, satisfying $\left\|\phi_{j}\right\|_{\infty} \geq c \lambda_{j}^{1 / 4}\|\phi\|_{2}$, complement the general upper bound $\left\|\phi_{j}\right\|_{\infty} \leq c \lambda_{j}^{1 / 2}\|\phi\|_{2}$ of Hörmander described below (1.2). Let $F(x), x=\left(x_{1}, x_{2}, x_{3}, x_{4}\right)$ be an integral quadratic form of signature $(3,1)$. Then $V=\{x \mid F(x)=-1\}$ is a two sheeted hyperboloid giving a model for $H^{3}$. Suppose that $\Gamma=O(F, \mathbb{Z})$ denotes the group of integral $4 \times 4$ matrices preserving $F$. Assume $F$ to be anisotropic over the rationals, that is $F(x) \neq 0$ for non-zero $x \in \mathbb{R}^{4}$. The examples of Rudnick and Sarnak are given as compact quotients $M=\Gamma \backslash H^{3}$. The proof rests upon Siegel's method of theta lifts from a Riemann surface. It would be very interesting to place these results in a more general geometric framework.

2. Quantum Unique Ergodicity. Suppose that $M$ is a compact Riemannian manifold. The Laplacian $\Delta$ of $M$ has pure point spectrum. Let $\phi_{k}$ denote an orthonormal basis of $L^{2} M$ consisting of eigenfunctions with eigenvalues $\lambda_{k}$, that is $\Delta \phi_{k}=-\lambda_{k} \phi_{k}$. The unit sphere bundle of the cotangent bundle is denoted by $S^{1} M$. Using the Friedrichs quantization, one associates to each function $a \in C^{\infty}\left(S^{1} M\right)$ the pseudodifferential operator $A=O p^{F}(a)$ of order zero. This particular quantization has the property that if $a \geq 0$, then $A \geq 0$ as on operator on $L^{2} M$. If $\phi_{k}$ is on eigenfunction of $\Delta$, then we define a measure $\mu_{k}$ by $\mu_{k}(a)=\left\langle O p^{F}(a) \phi_{k}, \phi_{k}\right\rangle$.

The measures $\mu_{k}$ are related in purpose to the supremum bounds of our previous section. These are two different methods for characterizing the concentration properties of eigenfunctions. For the manifolds of Theorem 1.4, which admit nondegenerate circle actions, there are eigenfunctions for which the $\mu_{k}$ concentrate on the unique maximal geodesic. The simplest example is provided by the equator of 
the standard sphere $S^{2}$. On the other hand, sinusoidal wave functions on the circle or flat torus exhibit no particular concentration.

Consider now the weak limits of the $\mu_{k}$ as $k \rightarrow \infty$. One possible weak limit is the normalized Liouville measure $d w$ on $S^{1} M$. Other limits may occur such as the singular measure concentrated on unit tangents to the equator in the two sphere. In general, one has

Lemma 2.1. Any weak limit of the $\mu_{k}$ is invariant under the geodesic flow on $S^{1} M$.

Proof. Let $G_{t}: S^{1} M \rightarrow S^{1} M$ denote the geodesic flow. If $A_{t}=$ $\exp (-i t \sqrt{-\Delta}) A \exp (i t \sqrt{-\Delta})$, then Egorov's theorem states that the difference $A_{t}-O p^{F}\left(a \circ G_{t}\right)$ is a pseudodifferential operator of order -1 . Consequently, $\lim _{k \rightarrow \infty}\left\langle O p^{F}\left(a \circ G_{t}\right) \phi_{k}, \phi_{k}\right\rangle=\lim _{k \rightarrow \infty}\left\langle O p^{F}(a) \phi_{k}, \phi_{k}\right\rangle$ and the result follows readily. 口

Moreover, the $\mu_{k}$ always converge on average to the Liouville measure. For each $\lambda>0$, let $N(\lambda)$ denote the number of eigenvalues of $\Delta$ that are less than $\lambda$. We have

Lemma 2.2. $\lim _{\lambda \rightarrow \infty} \frac{1}{N(\lambda)} \sum_{\lambda_{k} \leq \lambda} \int a d \mu_{k}=\int a d \omega$.

Proof. As $t \downarrow 0$, consider the heat equation asymptotics

$$
\sum_{k} e^{-t \lambda_{k}}\left\langle A \phi_{k}, \phi_{k}\right\rangle=\operatorname{Tr}\left(A e^{t \Delta}\right) \sim \operatorname{Tr}\left(e^{t \Delta}\right) \int_{M} a d \omega .
$$

Lemma 2.2 now follows from the Tauberian theorem of Karamata. $\square$

The preceding lemmas hold for any compact Riemannian manifold. No special hypotheses are needed concerning the geodesic flow. However, if the geodesic flow is ergodic there is a remarkable result. Theorem 2.3 below was first announced by Shnirelman [20]. Zelditch [23] subsequently published a proof for compact Riemann surfaces of constant negative curvature. A complete proof in the general case first appeared in the paper by Colin de Verdière [8]. For information concerning subsequent developments, the reader in referred to the survey article [24] and the references given there.

Theorem 2.3. Let $M$ be a compact Riemannian manifold. Assume that the geodesic flow on $S^{1} M$ is ergodic. Suppose that $\phi_{k}$ is an orthonomal basis, for $L^{2} M$, consisting of eigenfunctions on $\Delta$. Then for a subsequence $\phi_{k_{i}}$, having density one,

$$
\lim _{i \rightarrow \infty}\left\langle A \phi_{k_{i}}, \phi_{k_{i}}\right\rangle=\int_{S^{1} M} a d \omega
$$

Proof. Let $a_{T}(x)=\frac{1}{T} \int_{0}^{T}\left(a \circ G_{t}\right)(x) d t$. Since the geodesic flow is ergodic, we use the ergodic theorem to conclude that $\lim _{T \rightarrow \infty} a_{T}=\bar{a} \equiv \int_{S^{1} M} a d \omega$. If $N(\lambda)$ denotes the number of eigenvalues less than $\lambda$, then by Lemmas 2.1 and 2.2,

$$
\begin{aligned}
& \frac{1}{N(\lambda)} \sum_{\lambda_{k} \leq \lambda}\left|\mu_{k}(a)-\bar{a}\right| \leq \frac{1}{N(\lambda)} \sum_{\lambda_{k} \leq \lambda}\left|\mu_{k}(a)-\mu_{k}\left(a_{T}\right)\right| \\
& +\frac{1}{N(\lambda)} \sum_{\lambda_{k} \leq \lambda}\left|\mu_{k}\left(a_{T}\right)-\mu_{k}(\bar{a})\right| \leq \epsilon+\epsilon .
\end{aligned}
$$


For the additional details involving elementary number theory, the reader is referred to $[8]$.

The problem of quantum unique ergodicity is concerned with the sharpness of Theorem 2.3. If the conclusion of the theorem holds for all subsequences of eigenfunctions, then quantum unique ergodicity is said to hold. Otherwise there exist characteristic sequences of eigenfunctions where the weak limit of the associated measures $\mu_{k}$ is not the Liouville measure. The simplest candidate for such a limiting measure arises from concentration along a simple closed geodesic. Separation of variables produces germs of eigenfunctions which concentrate along an isolated hyperbolic geodesic. However, it is not known if a sequence of such germs can occur in a closed hyperbolic manifold. There do exist surfaces of revolution containing hyperbolic geodesics and exhibiting characteristic sequences of eigenfunctions. However, the rotational symmetry is incompatible with ergodic geodesic flow. So the problem of quantum unique ergodicity is global in nature. It is conceivable that quantum unique ergodicity may hold on all manifolds with ergodic geodesic flow. This would constitute a dramatic improvement of Theorem 2.3 .

In [14], the author approached the problem of quantum unique ergodicity by broadening the perspective. The basic idea is to show that Theorem 2.3 and its proof extend to a more general situation. For this generalized version of the theorem, exceptional sequences do occur on manifolds with ergodic geodesic flow. We begin with

Definition 2.4. An orthonormal basis $\phi_{k}$ of $L^{2} M$ consists of packets of eigenfunctions if there exists a sequence of spectral projectors $E_{k}$ for $\Delta$, supported on intervals of length at most $\delta>0$, so that $\phi_{k}=E_{k} \phi_{k}$. Here $\delta>0$ is independent of $k$.

By modifying the proof of Theorem 2.3, due to Colin de Verdière, we deduce

TheOREM 2.5. Let $M$ be a compact Riemannian manifold. Assume that the geodesic flow on $S^{1} M$ is ergodic. Suppose that $\phi_{k}$ is an orthonomal basis for $L^{2} M$, consisting of packets of eigenfunctions. Then, for a subsequence $\phi_{k_{i}}$, having density one,

$$
\lim _{i \rightarrow \infty}\left\langle A \phi_{k_{i}}, \phi_{k_{i}}\right\rangle=\int_{S^{1} M} a d \omega
$$

Theorem 2.5 is sharp. Namely, there exist surfaces supporting characteristic sequences of eigenfunction packets whose weak limits are different from the Liouville measure. The examples constructed in [14] are obtained by truncating manifolds of negative sectional curvature and finite volume. One attaches these together using long thin cylinders with curvature zero. A method of Burns and Gerber [7] is applied to show that the geodesic flow is ergodic. Quasimodes are constructed which concentrate microlocally on the transverse part of the cylinders. The eigenfunction packets are constructed by projecting the quasimodes back onto an interval of length $\delta$ and using the spectral theorem to show that the projection is non-trivial. Recall that a function $w$ is an $\epsilon$-accurate quasimode if there exists a real number $r$, so that $\|(\Delta+r) w\|_{2} \leq$ $\epsilon\|w\|_{2}$.

Consider a cylinder $[-L, L] \times S^{1}$, where $S^{1}$ has length $2 \pi \alpha$. Choose a cut-off function $\eta \in C_{0}^{\infty}[-L, L]$ so that $\eta(r)=1$ for $r \in[-L+1, L-1]$. the desired quasimodes are simply given by $w_{k}(r, \theta)=\eta(r) \exp (i k \theta / \alpha)$ with $r_{k}=k^{2} / \alpha^{2}$. Given $\epsilon>0$, if $L$ is sufficiently large, then $w_{k}$ is $\epsilon$-accurate. The quasimodes may be normalized by 
setting $u_{k}=w_{k} /\left\|w_{k}\right\|$. To show the microlocal concentration, we define a zero'th order pseudodifferential operator $A=(-\Delta+1)^{-1} \eta(r) \frac{\partial^{2}}{\partial r^{2}}$. The average value of the symbol of $A$ is non-zero but $\left(A u_{k}, u_{k}\right) \rightarrow 0$ as $k \rightarrow \infty$. Finally, let $E_{k}$ denote the spectral projector onto the interval $\left[r_{k}-\delta / 2, r_{k}+\delta / 2\right]$. For small $\epsilon \delta^{-1}$, the $E_{k} u_{k} /\left\|E_{k} u_{k}\right\|_{2}$ are the desired eigenfunction packets.

Given $\delta>0$, we may construct manifolds with $\operatorname{vol} M=1$ and such that the eigenvalues of the eigenfunction packets are concentrated on a interval of length $\delta$. For surfaces, Weyl's formula gives $N(\lambda) \sim c($ vol $M) \lambda$. So the average level spacing of the eigenvalues is normalized to $c^{-1}$. This suggests that the above examples may yield characteristic sequences of individual eigenfunctions. Thomas Spencer and Zelditch [25] have observed that it suffices to establish a uniform bound for the number of eigenfunctions appearing in each packet. This seems difficult since the best general estimate for the multiplicity is $c \lambda^{n-1 / 2}$, due to Hörmander [16]. Perhaps the estimate could be dramatically improved for manifolds with ergodic flow, or at least in these specific examples. In another direction, Burq and Zworski [6] showed that individual eigenfunctions cannot concentrate solely in the cylindrical part of the examples described above. Their results indicate that the above method of constructing quasimodes is the best possible.

Sarnak [19] conjectures that quantum unique ergodicity holds on all manifolds of negative sectional curvature. It is classical that negative sectional curvature implies ergodic geodesic flow. Although the case of variable negative curvature seems very open, there has been dramatic progress in the case of arithmetic manifolds having constant negative curvature. One assumes that the orthonormal bases of $L^{2} M$ are joint eigenfunctions of the Laplacian and of the Hecke operators. Rudnick and Sarnak [18] showed that scarring cannot occur along individual closed geodesics. Subsequently, Bourgain and Lindenstrauss [3 ] established positive entropy of arithmetic quantum limits. Building upon these previous results Lindenstrauss [17] proved quantum unique ergodicity in the case of surfaces.

3. Nodal Sets of Eigenfunctions. Suppose that $M$ is a connected compact Riemannian manifold with $C^{\infty}$ Riemannian metric. Let $\phi$ be a real eigenfunction of the Laplacian $\Delta$, associated to the metric, with eigenvalue $\lambda, \Delta \phi=-\lambda \phi$. If the metric and manifold were real analytic, then analytic hypoellipticity would yield unique continuation for the eigenfunctions of $\phi$. Remarkably, the work of Aronzajn [1 ] and Cordes [9], shows that even for $C^{\infty}$ metrics, the eigenfunction $\phi$ never vanishes to infinite order.

The nodal set $N$ is defined to be the set of points in $M$ where $\phi$ vanishes. Its singular part $S$ is the subset where both $\phi$ and its gradient $\nabla \phi$ vanish. If $M$ is two dimensional, the singular set consists of a finite number of points. Near each singular point, the nodal set is modeled upon the nodal set of an elementary harmonic function $\operatorname{Re}\left(z^{k}\right)$. If $n=\operatorname{dim} M>2$ there is no simple structure theorem for $N$ and $S$. However, the unique continuation theorem gives some estimates for the Hausdorff measures $\mathcal{H}$ :

LEMma 3.1. For all $\epsilon>0$, (i) $\mathcal{H}^{n-1+\epsilon} N=0$ and (ii) $H^{n-2+\epsilon} S=0$.

Proof. (i) Define $N_{k}=\left\{x \mid \phi(x)=\nabla \phi(x)=\ldots=\nabla^{k-1} \phi(x)=0\right.$ and $\left.\nabla^{k} \phi(x) \neq 0\right\}$, where $k \geq 1$. If $x \in N_{k}$, then there exists locally $g=\phi_{i_{i} i_{2} \ldots i_{k-1}}$ with $g(x)=0$ and $\nabla g(x) \neq 0$. One has a neighborhood $U_{x}$ with $U_{x} \cap N_{k}$ contained in the non-singular zero set of $g$. Choose a countable subcover of $M$.

(ii) Define $S_{k}=\left\{x \mid \phi(x)=\nabla \phi(x)=\cdots=\nabla^{k-1} \phi(x)=0\right.$ and $\left.\nabla^{k} \phi(x) \neq 0\right\}$, where $k \geq 2$. By rotation of normal coordinates centered at $x \in S_{k}$, we may as- 
sume there exists $g_{1}=\phi_{i_{i} i_{2} \ldots i_{k-2} j}$ defined locally with $\left(g_{1}\right)_{j}(x) \neq 0$. Since $\phi$ is an eigenfunction of $\Delta$, there must then exist $g_{2}=\phi_{i_{i} i_{2} \ldots i_{k-2} \ell}$ with $\left(g_{2}\right)_{\ell}(x) \neq 0$ and $\ell \neq j$. It follows that $S_{k}$ is locally contained in the intersection of two transverse submanifolds.

The next result is a prerequisite for the formulation of Yau's conjecture about the nodal set:

Lemma 3.2. For each fixed $\lambda, \mathcal{H}^{n-1} N$ is finite.

Proof. By part (ii) of Lemma 3.1, it suffices to prove that $\mathcal{H}^{n-1}(N-S)$ is finite. Since $\phi$ never vanishes to infinite order, the Malgrange preparation theorem shows that $N$ coincides locally with the zero set of a generalized polynomial $P=$ $x_{n}^{p}-\sum_{i=1}^{p-1} r_{i}\left(x_{1}, \ldots, x_{n-1}\right) x_{n}^{i}$, where $r_{i}$ are smooth functions vanishing at the origin. If $B$ is a hyperplane through the origin, then let $A=\left\{x \in N-S \mid \pi_{x}: T_{x}(N-S) \rightarrow B\right.$ satisfies $\left.\left|\pi_{x}\right|>\epsilon>0\right\}$. Fix $\epsilon>0$ and choose a finite collection $B_{i}, 1 \leq i \leq k$, of hyperplanes so that $N-S \subset \bigcup_{i=1}^{k} A_{i}$. Since the Malgrange preparation holds in a generic coordinate system, we have $\mathcal{H}_{l o c}^{n-1}(N-S) \leq c k p \epsilon^{-1}$. Now choose a finite subcover of the original compact manifold $M$.

Yau conjectures that for $C^{\infty}$ Riemannian manifolds $c_{1} \sqrt{\lambda} \leq \mathcal{H}^{n-1} N \leq c_{2} \sqrt{\lambda}$, where the constants $c_{1}$ and $c_{2}$ depend upon the Riemannian manifold $M$. This was proved by the author and Charles Fefferman [12] in the real analytic case. Some of the intermediate results proved there are also valid in the $C^{\infty}$ category.

A crucial ingredient in the proofs are refined Carleman inequalities. These inequalities are developed from Aronzajn's work [1], but careful attention is paid to dependence upon the parameter $\lambda$ in the eigenvalue equation $\Delta \phi=-\lambda \phi$. In the proposition below $(r, t)$ are geodesic spherical coordinates about a point and $\bar{r}$ is the geodesic distance for a conformally changed metric.

Proposition 3.3. Suppose that $u \in C_{0}^{\infty}(M)$ has support in $\delta / 2<r<h$, where $h<h_{0}$ is suitably small. Assume $\beta>a_{1} \sqrt{\lambda}+a_{2}$ with sufficiently large constants $a_{1}$ and $a_{2}$. Then

$$
\begin{aligned}
& \iint \bar{r}^{2(2-\beta)}|(\Delta+\lambda) u|^{2} r^{-1} d r d t \geq \\
& c_{1} \beta^{2} \iint \bar{r}^{2-2 \beta} u^{2} r^{-1} d r d t+c_{2} \delta \beta^{2} \iint \bar{r}^{-1-2 \beta} u^{2} r^{-1} d r d t
\end{aligned}
$$

To illustrate the utility of the Carleman inequality, we use it to show that the set where $\phi$ vanishes to infinite order is open. A connectedness argument then reveals that this set is empty. Let $\eta$ be a cut-off function satisfying $\eta \equiv 1$ in $\delta<r<h / 2$. 
Substitute $u=\eta \phi$ into the Carleman inequality. Then

$$
\begin{gathered}
\iint_{\{h / 2<r<h\}} \bar{r}^{2(2-\beta)}|(\Delta+\lambda) u|^{2} r^{-1} d r d t+ \\
\iint_{\{\delta / 2<r<\delta\}} \bar{r}^{2(2-\beta)}|(\Delta+\lambda) u|^{2} r^{-1} d r d t \geq c_{1} \beta^{2} \\
\iint_{\{h / 4<r<h / 2\}} \bar{r}^{2-2 \beta} \phi^{2} r^{-1} d r d t .
\end{gathered}
$$

If $\phi$ vanishes to infinite order at the origin, we may let $\delta \rightarrow 0$, to yield

$$
\iint_{\{h / 2<r<h\}} \bar{r}^{2(2-\beta)}|(\Delta+\lambda) u|^{2} r^{-1} d r d t \geq c_{1} \beta^{2} \iint_{\{h / 4<r<h / 2\}} \bar{r}^{2-2 \beta} \phi^{2} r^{-1} d r d t .
$$

Unless $\phi$ vanishes on a neighborhood of 0 , we obtain a contradiction when $\beta \rightarrow \infty$.

A more careful argument along the same lines yields a local alternative. If an eigenfunction vanishes to high order at a point, then either the eigenvalue $\lambda$ is large or the eigenfunction grows rapidly on concentric balls. The need for the second condition is apparent from consideration of harmonic polynomials vanishing to high order at the origin of $R^{n}$.

$$
\begin{aligned}
& \text { LEMMA 3.4. Let } \beta>a_{1} \sqrt{\lambda}+a_{2} \text { and } \beta>a_{3} \\
& \log \left(\max _{r \leq h}|\phi| / \max _{h / 10<r<h / 5}|\phi|\right) \text {. Then } \\
& \text { (i) } \max _{r \leq \delta}|\phi| \geq\left(c_{3} \delta\right)^{d_{1} \beta} \max _{h / 10 \leq r \leq h / 5}|\phi| \\
& \text { (ii) If }|\phi| \leq 1 \text { in } r \leq h \text { and } \max _{r \leq h / 5}|\phi| \geq \exp \left(-d_{2} \sqrt{\lambda}-c_{4}\right) \text {, then } \\
& \max _{r \leq h / 10}|\phi| \geq \exp \left(-d_{3} \sqrt{\lambda}-c_{5}\right)
\end{aligned}
$$

The example of harmonic polynomials again shows that local methods alone will not establish the next theorem. However, the global argument needed is of an elementary nature. Spherical harmonics on $S^{n}$ vanish to order $c \sqrt{\lambda}$. This illustrates the sharpness of

THEOREM 3.5. If $M$ is compact and $\Delta \phi=-\lambda \phi$, then $\phi$ vanishes to at most $\operatorname{order} c \sqrt{\lambda}$.

Proof. (Sketch) We normalize $\|\phi\|_{\infty}=1$ and choose $x_{0}$ with $\phi\left(x_{0}\right)=1$. For any $x \in M$, join $x_{0}$ to $x$ by a curve and subdivide to obtain points $x_{0}, x_{1}, x_{2}, \ldots, x_{l}=x$, with $x_{i+1} \in B\left(x_{i}, h / 10\right)$, a ball of radius $h / 10$ centered at $x_{i}$. Lemma 3.4, part(ii), and induction imply that

$$
\max _{B\left(x_{i}, h / 10\right)}|\phi| \geq \exp \left(-d_{i} \sqrt{\lambda}-e_{i}\right) .
$$

This argument uses a chain of overlapping balls. We now apply Lemma 3.4, part(i), with $\beta=d_{l} \sqrt{\lambda}+e_{l}$. 
The results cited above pertain primarily to the upper bound $\mathcal{H}^{n-1} N<c_{2} \sqrt{\lambda}$ in Yau's conjecture. For the lower bound $\mathcal{H}^{n-1} N>c_{1} \sqrt{\lambda}$, a prerequisite is the existence of sufficiently many nodal points. One may invoke

LEMMA 3.6. If $\Delta \phi=-\lambda \phi$, then $\phi$ vanishes at some point of each ball having radius at most $c \lambda^{-1 / 2}$.

Proof. Suppose that $\psi$ denotes the first Dirichlet eigenfunction of $B\left(p, c \lambda^{-1 / 2}\right)$. If $\phi$ is positive on this ball, then the ratio $g=\psi / \phi$ assumes an interior maximum. At this interior maximum point $0=g_{i}=\phi^{-1}\left(\psi_{i} \phi-\psi \phi_{i}\right)$ and $0 \geq \Delta g=\phi^{-2}(\Delta \psi \phi-\psi \Delta \phi) \geq$ $\phi^{-2}\left(-2^{-1} \lambda \psi \phi+\lambda \psi \phi\right)>0$, for $c$ sufficiently large. The result follows by contradiction to the hypothesis that $\phi$ is positive.

If $n=2$, it follows easily from the lemma that $\mathcal{H}^{1} N>c_{2} \sqrt{\lambda}$. This result is due to Brüning [5]. Recall that for surfaces $S$ is a finite set and $N-S$ is a smooth curve. Near each point of $S$, the nodal set has the same structure as $\operatorname{Re}\left(z^{k}\right)=0$, for some $k$. Closed loops inside $B\left(p, a \lambda^{-1 / 2}\right)$ are foreclosed by the monotonicity of Dirichlet eigenvalues, when $a$ is sufficiently small. Such arguments fail when $n>2$ and the conjecture $\mathcal{H}^{n-1} N>c_{2} \sqrt{\lambda}$ seems very difficult when $M$ is only smooth.

Yau's conjecture was proved for real analytic metrics in [10]:

THEOREM 3.7. If $M$ and its metric are real analytic, then $c_{1} \sqrt{\lambda} \leq \mathcal{H}^{n-1} N \leq$ $c_{2} \sqrt{\lambda}$

Proof. (Sketch) The motivating idea is that the eigenfunction $\phi$ behaves in many respects like a polynomial of order $c_{3} \sqrt{\lambda}$. Using the proof of analytic hypoellipticity one continues $\phi$ analytically from a coordinate chart $|x|<1$ in $R^{n}$ to a complex ball $|z|<2$ in $C^{n}$ and establishes the estimate

$$
\max _{|z|<1}|\phi(z)| \leq e^{c_{4} \sqrt{\lambda}} \max _{|x|<2}|\phi(x)| \text {. }
$$

Combining this with the method of Carleman inequalities gives

$$
\max _{|z|<1}|\phi(z)| \leq e^{c_{5} \sqrt{\lambda}} \max _{|x|<1 / 5}|\phi(x)| .
$$

In one complex variable, Jensen's lemma bounds the number of zeroes in terms of the growth. The upper bound $\mathcal{H}^{n-1} N \leq c_{2} \sqrt{\lambda}$ follows from the one variable result and an integral geometry argument.

The lower bound $\mathcal{H}^{n-1} N \geq c_{1} \sqrt{\lambda}$ uses Lemma 3.6 and one obtains a family of pairwise disjoint balls $B_{\nu}=B\left(x_{\nu}, d / \sqrt{\lambda}\right)$ covering a fixed portion of $M$. It is shown that $\mathcal{H}^{n-1}\left(B_{\nu} \cap N\right) \geq d_{1} \lambda^{-(n-1) / 2}$ for at least half of these balls. By the isoperimetric inequality, one need only show that the measure $B_{\nu}^{+}$of the set where $\phi$ is positive is commensurable to its complement $B_{\nu}^{-}$. This follows eventually from the growth estimate $(3.8)$ and the weak type $(1,1)$ inequality for the Hilbert transform.

Zelditch [24] has suggested that an asymptotic formula $\mathcal{H}^{1} N \sim c \sqrt{\lambda}$ may be obtained on real analytic manifolds with ergodic geodesic flow. Eigenfunctions of the form $\sin \left(k_{1} x_{1}\right) \sin \left(k_{2} x_{2}\right) \ldots \sin \left(k_{n} x_{n}\right)$ on $T^{n}=S^{1} \times S^{1} \times \ldots \times S^{1}$ show that asymptotics are precluded for general real analytic Riemannian manifolds.

For smooth Riemannian manifolds, Hardt and Simon [15] establish the upper bound $\mathcal{H}^{n-1} N \leq c_{3} \exp \left(c_{4} \sqrt{\lambda} \log \lambda\right)$. This provides a quantitative estimate in terms of $\lambda$, but this is far from the conjecture of Yau. If $n=2$, then $\mathcal{H}^{1} N \leq c_{5} \lambda^{3 / 4}$, according to [11]. An alternative proof of this result was subsequently given by Dong $[10]$. 


\section{REFERENCES}

[1] Aronszajn N., A unique continuation theorem for solutions of elliptic partial differential equations, J. Math. Pures Appl., 36 (1957), pp. 235-249.

[2] Bishop, R. and Crittenden, R., Geometry of Manifolds, Academic Press, N, Y., 1964.

[3] Bourgain, J. and Lindenstrauss, E., Entropy of quantum limits, Communications in Mathematical Physics, 233 (2003), pp. 153-171.

[4] Bourgain, J., Eigenfunction bounds for compact manifolds with integrable geodesic flows, IHES preprint, 1993.

[5] BRüning, J., Über Knoten von Eigenfunctionen des Laplace Beltrami Operator, Math. Z., 158 (1978), pp. 15-21.

[6] Burq, N. And Zworski, M., Geometric control in the presence of a black box, Journal of the American Mathematical Society, 17 (2004), pp. 443-471.

[7] Burns, K. And Gerber, M., Real analytic Bernoulli geodesic flows on $S^{2}$, Ergodic theory and dynamical systems, 9 (1989), pp. 27-45.

[8] Colin de Verdière, Y., Ergodicite et functions propre du laplacian, Communications in Mathematical Physics, 102 (1985), pp. 497-502.

[9] Cordes, H., Über die eindeutige Bestimmheit Lösungen elliptischer Differentialgleichungen durch anfangsvorgaben, Nachr. Akad. Wiss. Göttingen Math. Phys. Kl. II, 11 (1956), pp. 239-258.

[10] Dong, R.T., Nodal sets of eigenfunctions on Riemann surfaces, Journal of Differential Geometry, 36 (1992), pp. 493-506.

[11] Donnelly, H. And Fefferman, C., Nodal sets of eigenfunctions of the Laplacian on surfaces, Journal of the American Mathematical Society, 3 (1990), pp. 333-353.

[12] Donnelly, H. And Fefferman, C., Nodal sets of eigenfunctions on Riemannian manifolds, Inventiones Math., 93 (1988), pp. 161-183.

[13] Donnelly, H., Bounds for eigenfunctions of the Laplacian on compact Riemannian manifolds, J. Func. Anal., 187 (2001), pp. 247-261.

[14] Donnelly, H., Quantum unique ergodicity, Proceedings of the American Mathematical Society, 131 (2003), pp. 2945-2951.

[15] Hardt, R. And Simon, L., Nodal sets for solution of elliptic equations, Journal of Differential Geometry, 30 (1989), pp. 505-522.

[16] Hörmander, L., The spectral function of an elliptic operator, Acta Math., 121 (1968), pp. $193-218$.

[17] Lindenstrauss, E., Invariant measures and arithmetic quantum unique ergodicity, Annals of Mathematics, to appear.

[18] Rudnick, Z. And SARnAK, P., The behavior of eigenstates of arithmetic hyperbolic manifolds, Commun. Math. Phys., 161 (1994), pp. 195-213.

[19] Sarnak, P., Arithmetic quantum chaos, Blyth lecture series notes, University of Toronto, 1993.

[20] Shnirelman, A.I., Ergodic properties of eigenfunctions, Uspehi Mat. Nauk, 29 (1974), pp. $181-182$.

[21] SogGe, C., Concerning the $L^{p}$ norm of spectral clusters of second order elliptic operators on compact manifolds, J. Func. Anal., 77 (1988), pp. 123-168.

[22] Tотн, J.A. And Zelditch, S., Riemannian manifolds with uniformly bounded eigenfunctions, Duke Math. J., 111 (2002), pp. 97-132.

[23] Zelditch, S., Uniform distribution of eigenfunctions on a compact hyperbolic surface, Duke Mathematics Journal, 55 (1987), pp. 919-941.

[24] Zelditch, S., Asymptotics of Polynomials and Eigenfunctions, Proceedings of the ICM 2002, Vol II, pp. 733-742.

[25] Zelditch, S., Note on quantum unique ergodicity, Proceedings of the American Mathematical Society, 132 (2004), pp. 1869-1872. 
H. DONNELLY 